\title{
Exponential decay of correlations for the Rauzy-Veech-Zorich induction map.
}

\author{
Artur Avila* and Alexander Bufetov ${ }^{\dagger}$
}

October 30, 2018

\begin{abstract}
We prove exponential mixing for the Rauzy-Veech-Zorich induction map on the space of interval exchange transformations (Theorem 3).
\end{abstract}

\section{Introduction.}

This section mainly serves to fix notation. We follow the notation and the conventions of $[3]$.

\subsection{Rauzy operations $a$ and $b$.}

Let $\pi$ be a permutation on $m$ symbols. The permutation $\pi$ will always be assumed irreducible in the sense that $\pi\{1, \ldots, k\}=\{1, \ldots, k\}$ iff $k=m$. Rauzy operations $a$ and $b$ are defined by the formulas:

$$
\begin{aligned}
& a \pi(j)= \begin{cases}\pi j, & \text { if } j \leq \pi^{-1} m ; \\
\pi m, & \text { if } j=\pi^{-1} m+1 ; \\
\pi(j-1), & \text { other } j\end{cases} \\
& b \pi(j)= \begin{cases}\pi j, & \text { if } \pi j \leq \pi m ; \\
\pi j+1, & \text { if } \pi m<\pi j<m ; \\
\pi m+1, & \text { if } \pi j=m .\end{cases}
\end{aligned}
$$

These operations preserve irreducibility. The Rauzy class of a permutation $\pi$ is defined as the set of all permutations that can be obtained from $\pi$ by repeated application of the operations $a$ and $b$.

For $i, j=1, \ldots, m$, denote by $E_{i j}$ an $m \times m$ matrix of which the $i, j$-th element is equal to 1 , all others to 0 . Let $E$ be the $m \times m$-identity matrix.

${ }^{*}$ CNRS UMR 7599, Laboratoire de Probabilités et Modèles aléatoires, Université Pierre et Marie Curie-Boîte courrier 188, 75252-Paris Cedex 05, France

${ }^{\dagger}$ Department of Mathematics, Rice University 
Following Veech [6], we introduce the matrices

$$
\begin{gathered}
A(a, \pi)=\sum_{i=1}^{\pi^{-1}(m)} E_{i i}+E_{m, \pi^{-1} m+1}+\sum_{i=\pi^{-1} m+1}^{m} E_{i, i+1}, \\
A(b, \pi)=E+E_{m, \pi^{-1} m}
\end{gathered}
$$

For a vector $\lambda \in \mathbb{R}^{m}, \lambda=\left(\lambda_{1}, \ldots, \lambda_{m}\right)$, we write

$$
|\lambda|=\sum_{i} \lambda_{i}
$$

Let $\Delta_{m-1}$ be the unit simplex in $\mathbb{R}^{m}$ :

$$
\Delta_{m-1}=\left\{\lambda \in \mathbb{R}_{+}^{m}:|\lambda|=1\right\} .
$$

The space $\Delta(\mathcal{R})$ of interval exchange transformations, corresponding to a Rauzy class $\mathcal{R}$, is defined by the formula

$$
\Delta(\mathcal{R})=\Delta_{m-1} \times \mathcal{R} .
$$

Denote

$$
\Delta_{\pi}^{+}=\left\{\lambda \in \Delta_{m-1} \mid \lambda_{\pi^{-1} m}>\lambda_{m}\right\}, \quad \Delta_{\pi}^{-}=\left\{\lambda \in \Delta_{m-1} \mid \lambda_{m}>\lambda_{\pi^{-1} m}\right\},
$$

and

$$
\Delta^{+}=\cup_{\pi^{\prime} \in \mathcal{R}(\pi)} \Delta_{\pi^{\prime}}^{+}, \quad \Delta^{-}=\cup_{\pi^{\prime} \in \mathcal{R}(\pi)} \Delta_{\pi^{\prime}}^{-} .
$$

The Rauzy-Veech induction is a map

$$
\mathcal{T}: \Delta(\mathcal{R}) \rightarrow \Delta(\mathcal{R})
$$

defined by the formula

$$
\mathcal{T}(\lambda, \pi)= \begin{cases}\left(\frac{A(\pi, a)^{-1} \lambda}{\left|A(\pi,)^{-1} \lambda\right|}, a \pi\right), & \text { if } \lambda \in \Delta^{-} \\ \left(\frac{A(\pi, b)^{-1} \lambda}{\left|A(\pi, b)^{-1} \lambda\right|}, b \pi\right), & \text { if } \lambda \in \Delta^{+} .\end{cases}
$$

Veech [6] showed that the Rauzy-Veech induction has an absolutely continuous ergodic invariant measure on $\Delta(\mathcal{R})$; that measure is, however, infinite.

Following Zorich [9], define the function $n(\lambda, \pi)$ in the following way:

$$
n(\lambda, \pi)= \begin{cases}\inf \left\{k>0: \mathcal{T}^{k}(\lambda, \pi) \in \Delta^{-}\right\}, & \text {if } \lambda \in \Delta_{\pi}^{+} ; \\ \inf \left\{k>0: \mathcal{T}^{k}(\lambda, \pi) \in \Delta^{+}\right\}, & \text {if } \lambda \in \Delta_{\pi}^{-} .\end{cases}
$$

The Rauzy-Veech-Zorich induction is defined by the formula

$$
\mathcal{G}(\lambda, \pi)=\mathcal{T}^{n(\lambda, \pi)}(\lambda, \pi) .
$$

Theorem 1 (Zorich 9]) The map $\mathcal{G}$ has an ergodic invariant probability measure, absolutely continuous with respect to the Lebesgue measure class on $\Delta(\mathcal{R})$.

This invariant measure will be denoted by $\nu$. 


\subsection{Symbolic dynamics for the induction map.}

This subsection briefly describes the symbolic dynamics for the map $\mathcal{G}$. The notation follows 3 .

Consider the alphabet

$$
\mathcal{A}=\{(c, n, \pi) \mid c=a \text { or } b, n \in \mathbb{N}\}
$$

For $w_{1} \in \mathcal{A}, w_{1}=\left(c_{1}, n_{1}, \pi_{1}\right)$, we write $c_{1}=c\left(w_{1}\right), \pi_{1}=\pi\left(w_{1}\right), n_{1}=$ $n\left(w_{1}\right)$. For $w_{1}, w_{2} \in \mathcal{A}, w_{1}=\left(c_{1}, n_{1}, \pi_{1}\right), w_{2}=\left(c_{2}, n_{2}, \pi_{2}\right)$, define the function $B\left(w_{1}, w_{2}\right)$ in the following way: $B\left(w_{1}, w_{2}\right)=1$ if $c_{1}^{n_{1}} \pi_{1}=\pi_{2}$ and $c_{1} \neq c_{2}$ and $B\left(w_{1}, w_{2}\right)=0$ otherwise.

Introduce the space of words

$$
\mathcal{W}_{\mathcal{A}, B}=\left\{w=w_{1} \ldots w_{n} \mid w_{i} \in \mathcal{A}, B\left(w_{i}, w_{i+1}\right)=1 \text { for all } i=1, \ldots, n\right\} .
$$

For a word $w \in W_{\mathcal{A}, B}$, we denote by $|w|$ its length, i.e., the number of symbols in it; given two words $w(1), w(2) \in W_{\mathcal{A}, B}$, we denote by $w(1) w(2)$ their concatenation. Note that the word $w(1) w(2)$ need not belong to $W_{\mathcal{A}, B}$, unless a compatibility condition is satisfied by the last symbol of $w(1)$ and the first symbol of $w(2)$.

To each word assign the corresponding renormalization matrix as follows. For $w_{1} \in \mathcal{A}, w_{1}=\left(c_{1}, n_{1}, \pi_{1}\right)$, set

$$
A\left(w_{1}\right)=A\left(c_{1}, \pi_{1}\right) A\left(c_{1}, c_{1} \pi_{1}\right) \ldots A\left(c_{1}, c_{1}^{n_{1}-1} \pi_{1}\right)
$$

and for $w \in W_{\mathcal{A}, B}, w=w_{1} \ldots w_{n}$, set

$$
A(w)=A\left(w_{1}\right) \ldots A\left(w_{n}\right) .
$$

Words from $W_{\mathcal{A}, B}$ act on permutations from $\mathcal{R}$ : namely, if $w_{1} \in \mathcal{A}, w_{1}=$ $\left(c_{1}, n_{1}, \pi_{1}\right)$, then we set $w_{1} \pi_{1}=c_{1}^{n_{1}} \pi_{1}$. For permutations $\pi \neq \pi_{1}$, the symbol $w_{1} \pi$ is not defined. Furthermore, for $w \in W_{\mathcal{A}, B}, w=w_{1} \ldots w_{n}$, we write

$$
w \pi=w_{n}\left(w_{n-1}\left(\ldots w_{1} \pi\right) \ldots\right),
$$

assuming the right-hand side of the expression is defined. Finally, if $\pi^{\prime}=w \pi$, then we also write $\pi=w^{-1} \pi^{\prime}$.

We say that $w_{1} \in \mathcal{A}$ is compatible with $(\lambda, \pi) \in \Delta(\mathcal{R})$ if

1. either $\lambda \in \Delta_{\pi}^{+}, c_{1}=a$, and $a^{n_{1}} \pi_{1}=\pi$

2. or $\lambda \in \Delta_{\pi}^{-}, c_{1}=b$, and $b^{n_{1}} \pi_{1}=\pi$.

We say that a word $w \in W_{\mathcal{A}, B}, w=w_{1} \ldots w_{n}$ is compatible with $(\lambda, \pi)$ if $w_{n}$ is compatible with $(\lambda, \pi)$. We shall also sometimes say that $(\lambda, \pi)$ is compatible with $w$ instead of saying that $w$ is compatible with $(\lambda, \pi)$.

Now consider the sequence spaces

$$
\Omega_{\mathcal{A}, B}=\left\{\omega=\omega_{1} \ldots \omega_{n} \ldots \mid \omega_{n} \in \mathcal{A}, B\left(\omega_{n}, \omega_{n+1}\right)=1 \text { for all } n \in \mathbb{N}\right\}
$$


and

$\Omega_{\mathcal{A}, B}^{\mathbb{Z}}=\left\{\omega=\ldots \omega_{-n} \ldots \omega_{1} \ldots \omega_{n} \ldots \mid \omega_{n} \in \mathcal{A}, B\left(\omega_{n}, \omega_{n+1}\right)=1\right.$ for all $\left.n \in \mathbb{Z}\right\}$.

Denote by $\sigma$ the right shift on both these spaces. By a Theorem of Veech 6 , there exists a probability measure $\mathbb{P}$ on $\Omega_{\mathcal{A}, B}$ such that the dynamical systems $\left(\Omega_{\mathcal{A}, B}, \sigma, \mathbb{P}\right)$ and $(\Delta(\mathcal{R}), \mathcal{G}, \nu)$ are isomorphic. Indeed, let $w_{1} \in \mathcal{A}$ and define the set $\Delta\left(w_{1}\right)$ in the following way. If $w_{1}=\left(a, n_{1}, \pi_{1}\right)$, then

$$
\Delta\left(w_{1}\right)=\left\{(\lambda, \pi) \in \Delta^{-} \mid \exists \lambda^{\prime} \in \Delta_{a^{n_{1} \pi}}^{+} \text {such that } \lambda=\frac{A\left(w_{1}\right) \lambda^{\prime}}{\left|A\left(w_{1}\right) \lambda^{\prime}\right|}\right\} .
$$

If $w_{1}=\left(b, n_{1}, \pi_{1}\right)$, then

$$
\Delta\left(w_{1}\right)=\left\{(\lambda, \pi) \in \Delta^{+} \mid \exists \lambda^{\prime} \in \Delta_{b^{n_{1}} \pi}^{-} \text {such that } \lambda=\frac{A\left(w_{1}\right) \lambda^{\prime}}{\left|A\left(w_{1}\right) \lambda^{\prime}\right|}\right\} .
$$

In other words, for a letter $w_{1}=\left(c_{1}, n_{1}, \pi_{1}\right)$, the set $\Delta\left(w_{1}\right)$ is the set of all interval exchanges $(\lambda, \pi)$ such that, first, $\pi=\pi_{1}$, and, second, that the application of the map $\mathcal{G}$ to $(\lambda, \pi)$ results in $n_{1}$ applications of the Rauzy operation $c_{1}$.

The coding map $\Phi: \Delta(\mathcal{R}) \rightarrow \Omega_{\mathcal{A}, B}$ is given by the formula

$$
\Phi(\lambda, \pi)=\omega_{1} \ldots \omega_{n} \ldots \text { if } \mathcal{G}^{n}(\lambda, \pi) \in \Delta\left(\omega_{n}\right) .
$$

The $\mathcal{G}$-invariant smooth probability measure $\nu$ projects under $\Phi$ to a $\sigma$ invariant measure on $\Omega_{\mathcal{A}, B}$; probability with respect to that measure will be denoted by $\mathbb{P}$.

For $w \in W_{\mathcal{A}, B}, w=w_{1} \ldots w_{n}$, let

$$
C(w)=\left\{\omega \in \Omega_{\mathcal{A}, B} \mid \omega_{1}=w_{1}, \ldots, \omega_{n}=w_{n}\right\} .
$$

and

$$
\Delta(w)=\Phi^{-1}(C(w)) .
$$

W. Veech [6] has proved the following

Proposition 1 Let $\mathbf{q} \in W_{\mathcal{A}, B}$ be a word such that all entries of the matrix $A(\mathbf{q})$ are positive. Let $\omega \in \Omega_{\mathcal{A}, B}$ be a sequence having infinitely many occurrences of the word $\mathbf{q}$. Then the set $\Phi^{-1}(\omega)$ consists of one point.

We thus obtain an almost surely bijective symbolic dynamics for the map $\mathcal{G}$.

\subsection{Veech's space of zippered rectangles.}

A zippered rectangle associated to the Rauzy class $\mathcal{R}$ is a quadruple $(\lambda, h, a, \pi)$, where $\lambda \in \mathbb{R}_{+}^{m}, h \in \mathbb{R}_{+}^{m}, a \in \mathbb{R}^{m}, \pi \in \mathcal{R}$, and the vectors $h$ and $a$ satisfy the following equations and inequalities (one introduces auxiliary components $a_{0}=$ $h_{0}=a_{m+1}=h_{m+1}=0$, and sets $\left.\pi(0)=0, \pi^{-1}(m+1)=m+1\right)$ :

$$
h_{i}-a_{i}=h_{\pi^{-1}(\pi(i)+1)}-a_{\pi^{-1}(\pi(i)+1)-1}, i=0, \ldots, m
$$




$$
\begin{gathered}
h_{i} \geq 0, i=1, \ldots, m, a_{i} \geq 0, i=1, \ldots, m-1, \\
a_{i} \leq \min \left(h_{i}, h_{i+1}\right) \text { for } i \neq m, i \neq \pi^{-1} m, \\
a_{m} \leq h_{m}, a_{m} \geq-h_{\pi^{-1} m}, a_{\pi^{-1} m} \leq h_{\pi^{-1} m+1}
\end{gathered}
$$

The area of a zippered rectangle is given by the expression

$$
\lambda_{1} h_{1}+\cdots+\lambda_{m} h_{m}
$$

Following Veech, we denote by $\Omega(\mathcal{R})$ the space of all zippered rectangles, corresponding to a given Rauzy class $\mathcal{R}$ and satisfying the condition

$$
\lambda_{1} h_{1}+\cdots+\lambda_{m} h_{m}=1
$$

We shall denote by $x$ an individual zippered rectangle.

Veech [6] defines a flow $P^{t}$ and a map $\mathcal{U}$ on the space of zippered rectangles by the formulas:

$$
\begin{gathered}
P^{t}(\lambda, h, a, \pi)=\left(e^{t} \lambda, e^{-t} h, e^{-t} a, \pi\right) . \\
\mathcal{U}(\lambda, h, a, \pi)= \begin{cases}\left(A^{-1}(a, \pi) \lambda, A^{t}(a, \pi) h, a^{\prime}, a \pi\right), & \text { if }(\lambda, \pi) \in \Delta^{-} \\
\left(A^{-1}(b, \pi) \lambda, A^{t}(b, \pi) h, a^{\prime \prime}, b \pi\right), & \text { if }(\lambda, \pi) \in \Delta^{+},\end{cases}
\end{gathered}
$$

where

$$
\begin{array}{r}
a_{i}^{\prime}= \begin{cases}a_{i}, & \text { if } j<\pi^{-1} m, \\
h_{\pi^{-1} m}+a_{m-1}, & \text { if } i=\pi^{-1} m, \\
a_{i-1}, & \text { other } i .\end{cases} \\
a_{i}^{\prime \prime}= \begin{cases}a_{i}, & \text { if } j<m, \\
-h_{\pi^{-1} m}+a_{\pi^{-1} m-1}, & \text { if } i=m .\end{cases}
\end{array}
$$

The map $\mathcal{U}$ is invertible; $\mathcal{U}$ and $P^{t}$ commute $([6])$. Denote

$$
\tau(\lambda, \pi)=\left(\log \left(|\lambda|-\min \left(\lambda_{m}, \lambda_{\pi^{-1} m}\right)\right),\right.
$$

and for $x \in \Omega(\mathcal{R}), x=(\lambda, h, a, \pi)$, write

$$
\tau(x)=\tau(\lambda, \pi) .
$$

Now, following Veech [6], define

$$
\mathcal{Y}(\mathcal{R})=\{x \in \Omega(\mathcal{R})|| \lambda \mid=1\} .
$$

and

$$
\Omega_{0}(\mathcal{R})=\bigcup_{x \in \mathcal{Y}(\mathcal{R}), 0 \leq t \leq \tau(x)} P^{t} x
$$


$\Omega_{0}(\mathcal{R})$ is a fundamental domain for $\mathcal{U}$ and, identifying the points $x$ and $\mathcal{U} x$ in $\Omega_{0}(\mathcal{R})$, we obtain a natural flow, also denoted by $P^{t}$, on $\Omega_{0}(\mathcal{R})$. The space $\Omega_{0}(\mathcal{R})$ will be referred to as Veech's moduli space of zippered rectangles, and the flow $P^{t}$ as the Teichmüller flow on the space of zippered rectangles.

The space $\Omega(\mathcal{R})$ has a natural Lebesgue measure class and so does the transversal $\mathcal{Y}(\mathcal{R})$. Veech [6] has proved the following Theorem.

Theorem 2 (Veech 6]) There exists a measure $\mu_{\mathcal{R}}$ on $\Omega(\mathcal{R})$, absolutely continuous with respect to Lebesgue, preserved by both the map $\mathcal{U}$ and the flow $P^{t}$ and such that $\mu_{\mathcal{R}}\left(\Omega_{0}(\mathcal{R})\right)<\infty$.

\subsection{Symbolic representation for the flow $P^{t}$}

Following Zorich 9], define

$$
\begin{gathered}
\Omega^{+}(\mathcal{R})=\left\{x=(\lambda, h, a, \pi) \mid(\lambda, \pi) \in \Delta^{+}, a_{m} \geq 0\right\} . \\
\Omega^{-}(\mathcal{R})=\left\{x=(\lambda, h, a, \pi) \mid(\lambda, \pi) \in \Delta^{-}, a_{m} \leq 0\right\} \\
\mathcal{Y}^{+}(\mathcal{R})=\mathcal{Y}(\mathcal{R}) \cap \Omega^{+}(\mathcal{R}), \mathcal{Y}^{-}(\mathcal{R})=\mathcal{Y}(\mathcal{R}) \cap \Omega^{-}(\mathcal{R}), \mathcal{Y}^{ \pm}(\mathcal{R})=\mathcal{Y}^{+}(\mathcal{R}) \cup \mathcal{Y}^{-}(\mathcal{R}) .
\end{gathered}
$$

Take $x \in \mathcal{Y}^{ \pm}(\mathcal{R}), x=(\lambda, h, a, \pi)$, and let $\mathcal{F}(x)$ be the first return map of the flow $P^{t}$ on the transversal $\mathcal{Y}^{ \pm}(\mathcal{R})$. The map $\mathcal{F}$ is a lift of the map $\mathcal{G}$ to the space of zippered rectangles:

$$
\text { if } \mathcal{F}(\lambda, h, a, \pi)=\left(\lambda^{\prime}, h^{\prime}, a^{\prime}, \pi^{\prime}\right) \text {, then }\left(\lambda^{\prime}, \pi^{\prime}\right)=\mathcal{G}\left(\lambda^{\prime}, \pi^{\prime}\right) .
$$

Note that if $x \in \mathcal{Y}^{+}$, then $\mathcal{F}(x) \in \mathcal{Y}^{-}$, and if $x \in \mathcal{Y}^{-}$, then $\mathcal{F}(x) \in \mathcal{Y}^{+}$).

The map $\mathcal{F}$ preserves a natural absolutely continuous invariant measure on $\mathcal{Y}^{ \pm}(\mathcal{R})$ : indeed, since $\mathcal{Y}^{ \pm}(\mathcal{R})$ is a transversal to the flow $P^{t}$, the measure $\mu_{\mathcal{R}}$ induces an absolutely continuous measure $\bar{\nu}$ on $\mathcal{Y}^{ \pm}(\mathcal{R})$; since $\mu_{\mathcal{R}}$ is both $\mathcal{U}$ and $P^{t}$-invariant, the measure $\bar{\nu}$ is $\mathcal{F}$-invariant. Zorich [9] proved that the measure $\bar{\nu}$ is finite and ergodic for $\mathcal{F}$.

There exists a probability measure $\mathbb{P}$ on $\Omega_{\mathcal{A}, B}^{\mathbb{Z}}$ such that the dynamical system $\left(\mathcal{Y}^{ \pm}, \bar{\nu}, \mathcal{F}\right)$ is measurably isomorphic to the system $\left(\Omega_{\mathcal{A}, B}^{\mathbb{Z}}, \mathbb{P}, \sigma\right)[6$, 3]. The Teichmüller flow $P^{t}$ on the space $\Omega_{0}(\mathcal{R})$ is a suspension flow over the map $\mathcal{F}$ on $\mathcal{Y}^{ \pm}$. Identifying $\mathcal{Y}^{ \pm}$and $\Omega_{\mathcal{A}, B}^{\mathbb{Z}}$, we obtain a symbolic dynamics for the Teichmüller flow in the space of zippered rectangles.

\subsection{Formulation of the main result.}

We now fix a Rauzy class $\mathcal{R}$ and consider the space $\Delta(\mathcal{R})$ of interval exchange transformations corresponding to $\mathcal{R}$. We denote by $\mathcal{G}$ the Rauzy-Veech-Zorich induction map on $\mathcal{R}$ (for detailed definition, see [3]). As above, $\nu$ stands for the Zorich invariant measure of Theorem 1 The map $\mathcal{G}$ is not mixing: there exists a decomposition

$$
\Delta(\mathcal{R})=\Delta^{+}(\mathcal{R}) \bigcup \Delta^{-}(\mathcal{R}),
$$


such that $\mathcal{G}\left(\Delta^{+}(\mathcal{R})\right)=\Delta^{-}(\mathcal{R})$ and $\mathcal{G}\left(\Delta^{-}(\mathcal{R})\right)=\Delta^{+}(\mathcal{R})$.

The restriction of the map $\mathcal{G}^{2}$ on $\Delta^{+}(\mathcal{R})$ is, however, exact [3], and, consequently, strongly mixing.

We endow the space $\Delta(\mathcal{R})$ with the Hilbert metric $d$ by lifting the standard Hilbert metric on a simplex to $\Delta(\mathcal{R})$ (for a formal definition, see [3]), and, for an $\alpha$-Hölder function $\phi: \Delta(\mathcal{R}) \rightarrow \mathbb{R}$, by $|\phi|_{H_{\alpha}}$ we denote its $\alpha$-Hölder norm.

The main result of this note is the following.

Theorem 3 Let $\mathcal{G}: \Delta(\mathcal{R}) \rightarrow \Delta(\mathcal{R})$ be the Rauzy-Veech-Zorich induction map and let $\nu$ be the absolutely continuous invariant measure. Let $p>2$. Then, for any $\alpha>0$, there exist positive constants $C, \delta$ such that for any $\phi \in H_{\alpha} \cap$ $L_{p}\left(\Delta^{+}(\mathcal{R}), \nu\right)$ and $\psi \in L_{2}\left(\Delta^{+}(\mathcal{R}), \nu\right)$ we have

$$
\left|\int \phi \times \psi \circ \mathcal{G}^{2 n} d \nu-\int \phi d \nu \int \psi d \nu\right| \leq C \exp (-\delta n)\left(|\phi|_{H_{\alpha}}+|\phi|_{L_{p}}\right)\left(|\psi|_{L_{2}}\right) .
$$

Remark. A stretched-exponential estimate has been obtained in 3 .

\section{Proof of Theorem 3.}

\subsection{The tower method.}

The proof proceeds by the tower method of Lai-Sang Young [8].

First, following Veech, we consider a word $\mathbf{q}$ such that all entries of the renormalization matrix $A(\mathbf{q})$ are positive. An observation, going back to Veech [6], states that the first return map of $\mathcal{G}$ on $\Delta_{\mathbf{q}}$ of $\Delta(\mathcal{R})$ is uniformly expanding.

Now, for any $N \in \mathbb{N}$, introduce the set

$$
B(N, \mathbf{q})=\left\{(\lambda, \pi) \in \Delta_{\mathbf{q}} \mid \mathcal{G}^{n}(\lambda, \pi) \notin \Delta_{\mathbf{q}} \text { for all } n=1, \ldots, N\right\} .
$$

Lemma 1 There exist positive constants $C, \theta$, depending only on $\mathbf{q}$ and such that for any $N$ we have

$$
\mathbb{P}(B(N, \mathbf{q})) \leq C \theta^{N} .
$$

In view of the Lai-Sang Young Theorem 8, Lemma 1 implies Theorem 3 (note that the "uniform" version presented here follows from the statement in [8] by the Banach-Steinhaus Theorem).

It remains to establish Lemma 10 For the Teichmüller flow, exponential estimates for return times of the flow into compact sets already exist ([1, 3], [2]). In what follows, exponential estimate for the map are derived from those of the flow. More precisely, we shall compare the continuous return times for the flow with the discrete times for the induction map.

\subsection{Reduction to estimates on return times for the Te- ichmüller flow.}

Generally speaking, the continuous and the discrete return times are not comparable if the suspension function of the flow is bounded neither from above 
nor from below. Nonetheless, for return times into a set of the form $\Delta_{\mathbf{q}}$, where $q$ is a word such that all entries of the corresponding renormalization matrix are positive, we shall show that, indeed, the continuous and the discrete return times are comparable up to a multiplicative and an additive constant. This comparison will follow from Corollary 9 in $[\underline{3}$, which states that the norm of the Rauzy-Veech-Zorich renormalization matrix $A(w)$ grows exponentially in the length of a word $w \in W_{\mathcal{A}, B}$ and will allow us to conclude the proof of the Theorem. We proceed to precise statements.

Lemma 2 There exists a positive constant $C(\mathcal{R})$, depending only on the Rauzy class $\mathcal{R}$ such that for any $w \in W_{\mathcal{A}, B}$ we have

$$
\frac{|w|}{\log \|A(w)\|} \leq C(\mathcal{R}) .
$$

This statement immediately follows from Corollary 9 in [3] [it suffices to take the logarithm]. Now take $(\lambda, \pi) \in \Delta(\mathcal{R})$ and consider the corresponding symbolic expansion

$$
\Phi(\lambda, \pi)=\left(\omega_{1}, \ldots, \omega_{n}, \ldots\right) .
$$

Set

$$
n_{\mathbf{q}}(\lambda, \pi)=\min \left\{n>0 \mid \mathcal{G}^{n}(\lambda, \pi) \in \Delta_{\mathbf{q}}\right) .
$$

Introduce the word $w_{\mathbf{q}}(\lambda, \pi)$ by the formula

$$
w=\omega_{1} \ldots \omega_{n_{\mathbf{q}}}
$$

and denote

$$
\eta_{\mathbf{q}}(\lambda, \pi)=\log \left\|A\left(w_{\mathbf{q}}(\lambda, \pi)\right)\right\| .
$$

and

$$
\tau_{\mathbf{q}}(\lambda, \pi)=\log \left|A\left(w_{\mathbf{q}}(\lambda, \pi)\right) \lambda\right| .
$$

By definition, $\tau_{q}(\lambda, \pi)$ is the time it takes a zippered rectangle with base $(\lambda, \pi)$ under the Teichmüller flow to reach $\Delta_{q}$. Note that, by definition, $\eta_{q}>$ $\tau_{q}$. Generally speaking, these quantities are not comparable: for some interval exchanges $(\lambda, \pi)$, it may happen that $\tau_{\mathbf{q}}(\lambda, \pi)$ is very small while $\eta_{\mathbf{q}}(\lambda, \pi)$ may be large. Nonetheless, we have

Proposition 2 There exists a constant $C_{11}(q)$ such that for all $(\lambda, \pi) \in \Delta_{q}$ we have

$$
\eta_{\mathbf{q}}(\lambda, \pi)-\tau_{\mathbf{q}}(\lambda, \pi)<C_{11}(q)
$$

This follows from the fact that if $(\lambda, \pi) \in \Delta_{\mathbf{q}}$, then there exists a constant $C_{21}(\mathbf{q})$ such that

$$
\frac{\lambda_{i}}{\lambda_{j}} \leq C_{21}(\mathbf{q})
$$

and then for any matrix $A$ with nonnegative entries we have 


$$
\frac{|A \lambda|}{\|A\|} \geq C_{31}(\mathbf{q})
$$

for some constant $C_{31}(\mathbf{q})$, depending only on $\mathbf{q}$, and the Proposition is proved.

We now complete the Proof of Lemma There exists $\epsilon>0$, depending only on $\mathbf{q}$ and such that we have the following estimate

$$
\int_{\Delta} \exp \left(\epsilon \tau_{\mathbf{q}}\right) d \mathbb{P}<\infty .
$$

This estimate, extending earlier estimates of J. Athreya on returns of the Teichmüller flow into compact sets, is obtained in [3], $\S 11$ and also, independently, in [2], 66 .

Proposition 2 now yields

$$
\int_{\Delta} \exp \left(\epsilon \eta_{\mathbf{q}}\right) d \mathbb{P}<\infty,
$$

which, by Lemma 2 implies, for some $\tilde{\epsilon}$, depending only on $\mathbf{q}$, the estimate:

$$
\int_{\Delta} \exp \left(\tilde{\epsilon} n_{\mathbf{q}}\right) d \mathbb{P}<\infty,
$$

whence, finally, we obtain

$$
\mathbb{P}(B(N, \mathbf{q})) \leq C \theta^{N},
$$

the result of Lemma 1 Theorem 3 is proved completely.

Acknowledgements. We are deeply grateful to Giovanni Forni for our many stimulating discussions and to the referee for the careful reading of the manuscript and suggestions on improving the presentation. We gratefully acknowledges the hospitality of the Institut Henri Poincaré in Paris and Institut des Mathématiques in Luminy, where part of this work was done. In summer 2005 A.B. was a Clay Liftoff Fellow. A.B.'s research is partially supported by the National Science Foundation under grant DMS0604386. This research was partially conducted during the period A.A. served as a Clay Research Fellow.

\section{References}

[1] J. Athreya, Quantitative recurrence and large deviations for Teichmüller geodesic flow. Geometriae Dedicata 119 (2006), no. 1, 121-140.

[2] A. Avila, S. Gouëzel, J.-C. Yoccoz, Exponential mixing for the Teichmüller flow, preprint, www.arxiv.org. To appear in Pub. Math. IHES.

[3] Alexander I. Bufetov, Decay of Correlations for the Rauzy-Veech-Zorich Induction Map on the Space of Interval Exchange Transformations and the Central Limit Theorem for the Teichmüller Flow on the Moduli Space of Abelian Differentials, J. Amer. Math. Soc. 19 (2006), 579-623. 
[4] H. Masur, Interval exchange transformations and measured foliations. Ann. of Math. (2) 115 (1982), no. 1, 169-200.

[5] G.Rauzy, Échanges d'intervalles et transformations induites. Acta Arith. 34, (1979), no. 4, 315-328.

[6] William Veech, Gauss measures for transformations on the space of interval exchange maps, Annals of Mathematics, 15(1982), 201-242.

[7] W.Veech, Interval exchange transformations. J. Analyse Math. 33 (1978), $222-272$.

[8] Lai-Sang Young, Recurrence times and rates of mixing, Israel J. Mathematics, 110(1999), 153-188.

[9] Anton Zorich, Finite Gauss measure on the space of interval exchange transformations. Lyapunov exponents. Ann. Inst. Fourier (Grenoble) 46 (1996), no. $2,325-370$. 